\title{
ON SYMMETRIES AND INVARIANT SOLUTIONS OF A COUPLED KdV SYSTEM WITH VARIABLE COEFFICIENTS
}

\author{
K. SINGH AND R. K. GUPTA \\ Received 22 February 2005 and in revised form 18 October 2005
}

We investigate symmetries and reductions of a coupled KdV system with variable coefficients. The infinitesimals of the group of transformations which leaves the KdV system invariant and the admissible forms of the coefficients are obtained using the generalized symmetry method based on the Fréchet derivative of the differential operators. An optimal system of conjugacy inequivalent subgroups is then identified with the adjoint action of the symmetry group. For each basic vector field in the optimal system, the KdV system is reduced to a system of ordinary differential equations, which is further studied with the aim of deriving certain exact solutions.

\section{Introduction}

The last few decades have witnessed a tremendous growth in research interests related to the applications of group theoretical methods while deriving exact solutions to a wide range of problems describing nonlinear phenomena. The most effective methods, for finding the symmetry reduction and constructing exact solutions, include the classical Lie approach $[7,16,21,26]$, the nonclassical approach $[2,6,13,25]$, the direct method $[11,12]$, the modified direct method [22], the generalized conditional symmetry method $[14,31]$, nonlocal symmetry method $[1,8]$, the truncated Painlevé approach $[9,24]$, the transformation method $[18,19]$, and the ansatz-based methods $[10,17,20,27,30]$. There are in fact, many different directions of the mathematical and physical theory to concern their exact solutions and various properties.

Herein, we study the symmetries and reductions of a coupled KdV system, with variable coefficients, of the form

$$
\begin{gathered}
u_{t}+\alpha_{1}(t) u u_{x}+\beta_{1}(t) v v_{x}+\gamma_{1}(t) u_{x x x}=0 \\
v_{t}+\alpha_{2}(t) v u_{x}+\beta_{2}(t) u v_{x}+\gamma_{2}(t) v_{x x x}=0 .
\end{gathered}
$$

Equations (1.1) are an extension of the KdV system examined by Zhou et al. [32] for periodic wave solutions using the $F$-expansion method, an overall generalization of the Jacobi elliptic function expansion method. The constant coefficient version of (1.1) was 
earlier discussed by Lei et al. [28], wherein the author had derived the classical periodic solutions using Fourier expansion on an incomplete basis.

To determine the underlying symmetry group for (1.1), we utilize a method which is based on the Fréchet derivative of the differential operators associated with the system (1.1). The technique has earlier been used to obtain the exact solutions of various nonlinear partial differential equations (refer to [3, 4, 5, 23]). However, for the sake of completeness, and in order to help the reader relate it to the familiar one-parameter group of transformations, we provide here some basic knowledge about the approach.

Let the system (1.1) be defined in terms of the nonlinear operators $N_{1}$ and $N_{2}$ as follows:

$$
\begin{aligned}
& N_{1}(u, v) \equiv u_{t}+\alpha_{1}(t) u u_{x}+\beta_{1}(t) v v_{x}+\gamma_{1}(t) u_{x x x}=0 \\
& N_{2}(u, v) \equiv v_{t}+\alpha_{2}(t) v u_{x}+\beta_{2}(t) u v_{x}+\gamma_{2}(t) v_{x x x}=0
\end{aligned}
$$

Next, we define an operator called a symmetry operator for the system (1.2) given by

$$
\bar{S}=\left(S_{1}, S_{2}\right),
$$

where

$$
\begin{aligned}
& S_{1}(u) \equiv A(\bar{X}, \bar{\eta}) \frac{\partial u}{\partial t}+B(\bar{X}, \bar{\eta}) \frac{\partial u}{\partial x}+C_{1}(\bar{X}, \bar{\eta}), \\
& S_{2}(v) \equiv A(\bar{X}, \bar{\eta}) \frac{\partial v}{\partial t}+B(\bar{X}, \bar{\eta}) \frac{\partial v}{\partial x}+C_{2}(\bar{X}, \bar{\eta})
\end{aligned}
$$

with $\bar{X}=(t, x), \bar{\eta}=(u, v)$.

The Fréchet derivative of $\bar{N}(\bar{\eta})=\left(N_{1}, N_{2}\right)$ in the direction of $\bar{\eta}_{1}=\left(u_{1}, v_{1}\right)$ is given by

$$
\bar{F}\left(\bar{N}, \bar{\eta}, \bar{\eta}_{1}\right)=\left.\frac{d}{d \varepsilon}\left[\bar{N}\left(\bar{\eta}+\varepsilon \bar{\eta}_{1}\right)\right]\right|_{\varepsilon=0} .
$$

For invariance of (1.1), we require that the Fréchet derivative (1.5) must vanish on the solution set $\Gamma$ of (1.1) in the direction of the symmetry operator $\bar{S}$. That is, we must have

$$
\left.\bar{F}(\bar{N}, \bar{\eta}, \bar{S})\right|_{\Gamma}=\bar{O} .
$$

The associated Lie algebra of infinitesimal symmetries of (1.1) is then the set of vector fields of the form

$$
V=A(\bar{X}, \bar{\eta}) \frac{\partial}{\partial t}+B(\bar{X}, \bar{\eta}) \frac{\partial}{\partial x}-C_{1}(\bar{X}, \bar{\eta}) \frac{\partial}{\partial u}-C_{2}(\bar{X}, \bar{\eta}) \frac{\partial}{\partial v} .
$$

Or, equivalently the one-parameter group of point transformations of (1.1) is as follows:

$$
\begin{aligned}
t^{*} & =t+\varepsilon A(\bar{X}, \bar{\eta})+O\left(\varepsilon^{2}\right), \\
x^{*} & =x+\varepsilon B(\bar{X}, \bar{\eta})+O\left(\varepsilon^{2}\right), \\
\bar{\eta}^{*} & =\bar{\eta}-\varepsilon \bar{C}(\bar{X}, \bar{\eta})+O\left(\varepsilon^{2}\right),
\end{aligned}
$$

where $\bar{C}=\left(C_{1}, C_{2}\right) \bar{\eta}^{*}=\left(u^{*}, v^{*}\right)$. 
The advantage in our approach is that it, not only furnishes the group infinitesimals comparatively easily but, also often renders symmetries more generalized than the classical Lie method (refer to [4], e.g.). It is precisely due to this fact that we prefer to call the said approach the generalized symmetry method. Besides demonstrating the importance and efficacy of the method, the motivation for the present study lies in the physical importance of the model KdV system (1.1) and the need to have some exact solutions. To have an insight, the explicit analytic solutions of the system (1.1) may enable one to better understand the physical phenomena which it describes. The exact solutions, which are accurate and explicit, may help physicists and engineers to discuss and examine the sensitivity of the model with variable coefficients to several important physical parameters. To our knowledge, a detailed analysis that leads to an exact analytic solution for (1.1) has not been performed and is therefore desirable.

The paper has been organized as follows. Section 2 is entirely devoted to showing how the powerful generalized symmetry method can be used to generate various symmetries of the KdV system. In Section 3, we present the reduced systems of ordinary differential equations (ODEs) and their exact solutions. The final section contains the discussion and concluding remarks.

\section{The symmetry group and the optimal system}

The method for determining the symmetry group of (1.1) mainly consists of finding the coefficients $A, B, C_{1}$, and $C_{2}$ in the two symmetry operators $S_{1}$ and $S_{2}$ as defined by (1.4). These coefficients are to be determined from the invariance condition (1.6). Accordingly, we first find the Fréchet derivative

$$
\bar{F}\left(\bar{N}, \bar{\eta}, \bar{\eta}_{1}\right)=\left(F_{1}\left(N_{1}, \bar{\eta}, \bar{\eta}_{1}\right), F_{2}\left(N_{2}, \bar{\eta}, \bar{\eta}_{1}\right)\right)
$$

where

$$
\begin{aligned}
& F_{1}\left(N_{1}, \bar{\eta}, \bar{\eta}_{1}\right)=\left.\frac{d}{d \varepsilon}\left[N_{1}\left(\bar{\eta}+\varepsilon \bar{\eta}_{1}\right)\right]\right|_{\varepsilon=0}, \\
& F_{2}\left(N_{2}, \bar{\eta}, \bar{\eta}_{1}\right)=\left.\frac{d}{d \varepsilon}\left[N_{2}\left(\bar{\eta}+\varepsilon \bar{\eta}_{1}\right)\right]\right|_{\varepsilon=0} .
\end{aligned}
$$

With the help of (2.2) the Fréchet derivatives are obtained and then $\bar{\eta}_{1}$ is substituted by $\bar{S}=\left(S_{1}, S_{2}\right)$ in order to evaluate them in the direction of the symmetry operator. This leads to the following

$$
\begin{aligned}
& F_{1}\left(N_{1}, \bar{\eta}, \bar{S}\right)=\left[S_{1}\right]_{t}+\alpha_{1}\left[S_{1}\right] u_{x}+\alpha_{1}\left[S_{1}\right]_{x} u+\beta_{1} v\left[S_{2}\right]_{x}+\beta_{1}\left[S_{2}\right] v_{x}+\gamma_{1}\left[S_{1}\right]_{x x x}, \\
& F_{2}\left(N_{2}, \bar{\eta}, \bar{S}\right)=\left[S_{2}\right]_{t}+\alpha_{2}\left[S_{2}\right] u_{x}+\alpha_{2}\left[S_{1}\right]_{x} v+\beta_{2} u\left[S_{2}\right]_{x}+\beta_{2}\left[S_{1}\right] v_{x}+\gamma_{2}\left[S_{2}\right]_{x x x} .
\end{aligned}
$$

For invariance of system (1.1), the following conditions must be satisfied:

$$
\begin{aligned}
& \left.F_{1}\left(N_{1}, \bar{\eta}, \bar{S}\right)\right|_{\left(N_{1}, N_{2}\right)=\bar{O}}=0, \\
& \left.F_{2}\left(N_{2}, \bar{\eta}, \bar{S}\right)\right|_{\left(N_{1}, N_{2}\right)=\bar{O}}=0 .
\end{aligned}
$$


Equations (2.4), when expanded, result in the polynomial expressions in various partial derivatives of $u(t, x)$ and $v(t, x)$ with respect to the spatial variable. The calculations involved are tedious, however, to keep the interest of the reader we list here a much simplified set of determining equations for the group infinitesimals $A, B, C_{1}$, and $C_{2}$ which we get from (2.4a) after equating the coefficients of various derivative terms to zero:

$$
\begin{gathered}
A_{x}=A_{u}=A_{v}=0 \\
B_{u}=B_{v}=0 \\
C_{1 u u}=C_{1 v v}=C_{1 u v}=C_{1 x v}=0 \\
-\gamma_{1} A_{t}-A \gamma_{1 t}+3 \gamma_{1} B_{x}=0 \\
\left(\gamma_{1}-\gamma_{2}\right) C_{1 v}=0 \\
3 \gamma_{1}\left(B_{x x}+C_{1 x u}\right)=0 \\
-\alpha_{1} u A_{t}-A \alpha_{1 t} u+B_{t}-\alpha_{2} v C_{1 v}+\alpha_{1} C_{1}+\alpha_{1} u B_{x}+\beta_{1} v C_{2 u}+\gamma_{1} B_{x x x}+3 \gamma_{1} C_{1 x x u}=0 \\
-\beta_{1} v A_{t}-A \beta_{1 t} v-\beta_{1} v C_{1 u}-\beta_{2} u C_{1 v}+\alpha_{1} u C_{1 v}+\beta_{1} C_{2}+\beta_{1} v B_{x}+\beta_{1} v C_{2 v}=0 \\
C_{1 t}+\alpha_{1} u C_{1 x}+\beta_{1} v C_{2 x}+\gamma_{1} C_{1 x x x}=0 .
\end{gathered}
$$

Similarly, (2.4b) brings in the following additional equations. It is being mentioned here that these equations have been obtained keeping in view the consequences on the infinitesimals as effected by the set of equations (2.5):

$$
\begin{gathered}
C_{2 u u}=C_{2 v v}=C_{2 u v}=C_{2 x u}=0, \\
\left(\gamma_{2}-\gamma_{1}\right) C_{2 u}=0, \\
3 \gamma_{2}\left(B_{x x}+C_{2 x v}\right)=0, \\
-\gamma_{2} A_{t}-A \gamma_{2 t}+3 \gamma_{2} B_{x}=0, \\
-\alpha_{2} v A_{t}-A \alpha_{2 t} v-\alpha_{1} u C_{2 u}-\alpha_{2} v C_{2 v}+\alpha_{2} C_{2}+\alpha_{2} v B_{x}+\alpha_{2} v C_{1 u}+\beta_{2} u C_{2 u}=0, \\
-\beta_{2} u A_{t}-A \beta_{2 t} u+B_{t}-\beta_{1} v C_{2 u}+\alpha_{2} v C_{1 v}+\beta_{2} C_{1}+\beta_{2} u B_{x}=0, \\
C_{2 t}+\alpha_{2} v C_{1 x}+\beta_{2} u C_{2 x}+\gamma_{2} C_{2 x x x}=0 .
\end{gathered}
$$

The two sets of equations (2.5) and (2.6) are further simplified to the extent possible and we eventually arrive at the following version of the determining equations:

$$
\begin{gathered}
A=A(t), \quad B=B_{1}(t) x+B_{2}(t), \quad C_{1}=\phi_{1}(t) u+\phi_{2}(x, t), \\
C_{2}=\varphi(t) v, \quad \phi_{1 t}+\alpha_{1} \phi_{2 x}=0, \quad \phi_{2 t}+\gamma_{1} \phi_{2 x x x}=0, \\
\varphi_{t}+\alpha_{2} \phi_{2 x}=0, \quad B_{1 t} x+B_{2 t}+\alpha_{1} \phi_{2}=0, \quad B_{1 t} x+B_{2 t}+\beta_{2} \phi_{2}=0, \\
-\alpha_{1} A_{t}-A \alpha_{1 t}+\alpha_{1} \phi_{1}+\alpha_{1} B_{1}=0, \quad-\gamma_{1} A_{t}-A \gamma_{1 t}+3 \gamma_{1} B_{1}=0, \\
-\gamma_{2} A_{t}-A \gamma_{2 t}+3 \gamma_{2} B_{1}=0, \quad-\beta_{1} A_{t}-A \beta_{1 t}-\beta_{1} \phi_{1}+2 \beta_{1} \varphi+\beta_{1} B_{1}=0, \\
-\alpha_{2} A_{t}-A \alpha_{2 t}+\alpha_{2} B_{1}+\alpha_{2} \phi_{1}=0, \quad-\beta_{2} A_{t}-A \beta_{2 t}+\beta_{2} B_{1}+\beta_{2} \phi_{1}=0 .
\end{gathered}
$$


Equations (2.7) enable us to derive the infinitesimals $A, B, C_{1}$, and $C_{2}$, along with the admissible forms of the various coefficient functions of system (1.1). Without presenting any calculations, we provide the results obtained as follows:

$$
\begin{gathered}
A=\frac{1}{\alpha_{1}(t)}\left[\left(a_{1}+a_{2}\right) \int \alpha_{1}(t) d t+a_{4}\right], \\
B=a_{1} x+a_{5}, \quad C_{1}=a_{2} u, \quad C_{2}=a_{3} v,
\end{gathered}
$$

where $a_{j}, j=1,2, \ldots, 5$ are arbitrary constants. The functions $\alpha_{1}(t), \beta_{1}(t), \gamma_{1}(t), \alpha_{2}(t)$, $\beta_{2}(t)$, and $\gamma_{2}(t)$ are governed by the following relations:

$$
\begin{gathered}
\frac{d}{d t}\left[A(t) \beta_{1}(t)\right]+\left(a_{2}-2 a_{3}-a_{1}\right) \beta_{1}(t)=0, \\
\frac{d}{d t}\left[A(t) \gamma_{i}(t)\right]-3 a_{1} \gamma_{i}(t)=0, \quad i=1,2, \\
\alpha_{2}(t)=k_{1} \alpha_{1}(t), \quad \beta_{2}(t)=k_{2} \alpha_{1}(t),
\end{gathered}
$$

where $k_{1}$ and $k_{2}$ are arbitrary constants. The symmetries under which (1.1) is invariant can be spanned by the following five linearly independent infinitesimal generators:

$$
\begin{gathered}
V_{1}=\left[\frac{1}{\alpha_{1}(t)} \int \alpha_{1}(t) d t\right] \frac{\partial}{\partial t}+x \frac{\partial}{\partial x}, \quad V_{2}=\left[\frac{1}{\alpha_{1}(t)} \int \alpha_{1}(t) d t\right] \frac{\partial}{\partial t}-u \frac{\partial}{\partial u}, \\
V_{3}=-v \frac{\partial}{\partial v}, \quad V_{4}=\frac{1}{\alpha_{1}(t)} \frac{\partial}{\partial t}, \quad V_{5}=\frac{\partial}{\partial x} .
\end{gathered}
$$

Using these generators one can obtain a reduction of (1.1) to a system of ODEs after getting the similarity variable and the form by solving the characteristic equations

$$
\frac{d t}{A}=\frac{d x}{B}=-\frac{d u}{C_{1}}=-\frac{d v}{C_{2}}
$$

In general, one may obtain the reduced system of ODEs from any linear combination of generators $V_{j}, j=1,2, \ldots, 5$. Since there exist infinite possibilities for such combinations, a systematic procedure to classify these reductions is based on the property that the transformations of the symmetry group will transform solutions of (1.1) into other solutions. Therefore, we classify the symmetry algebra of the KdV system into conjugacy inequivalent subalgebra under the adjoint action of the symmetry group. We thus deduce the following basic fields which form an optimal system for the KdV system, and from which every other solution can be derived:

(i) $V_{1}+a V_{2}+b V_{3}$,

(ii) $V_{2}+c V_{3}$,

(iii_1) $V_{2}+d V_{3}+V_{5}$

(iii_2) $V_{2}+d V_{3}-V_{5}$,

(iv) $V_{3}+\mu V_{4}$, 
Table 2.1

\begin{tabular}{|c|c|c|c|}
\hline $\begin{array}{l}\text { Type of } \\
\text { essential } \\
\text { field }\end{array}$ & $\begin{array}{l}\text { Similarity variable } \\
\xi\end{array}$ & $\begin{array}{l}\text { Similarity solution } \\
\qquad(u, v)\end{array}$ & $\begin{array}{l}\text { Forms of coefficient } \\
\text { functions }\end{array}$ \\
\hline (i) & $x\left[\int \alpha_{1}(t) d t\right]^{1 /(1+a)}$ & $\begin{array}{l}{\left[\int \alpha_{1}(t) d t\right]^{-a /(1+a)} F(\xi)} \\
{\left[\int \alpha_{1}(t) d t\right]^{-b /(1+a)} G(\xi)}\end{array}$ & $\begin{array}{l}\beta_{1}(t)=k_{3} \alpha_{1}(t)\left[\int \alpha_{1}(t) d t\right]^{2(b-a) /(1+a)} \\
\alpha_{2}(t)=k_{1} \alpha_{1}(t), \beta_{2}(t)=k_{2} \alpha_{1}(t) \\
\gamma_{i}(t)=\sigma_{i} \alpha_{1}(t)\left[\int \alpha_{1}(t) d t\right]^{(2-a) /(1+a)} \\
\quad i=1,2\end{array}$ \\
\hline (ii) & $x$ & $\begin{array}{l}{\left[\int \alpha_{1}(t) d t\right]^{-1} F(\xi),} \\
{\left[\int \alpha_{1}(t) d t\right]^{-c} G(\xi)}\end{array}$ & $\begin{array}{l}\beta_{1}(t)=k_{3} \alpha_{1}(t)\left[\int \alpha_{1}(t) d t\right]^{2(c-1)}, \\
\alpha_{2}(t)=k_{1} \alpha_{1}(t), \beta_{2}(t)=k_{2} \alpha_{1}(t), \\
\gamma_{i}(t)=\sigma_{i} \alpha_{1}(t)\left[\int \alpha_{1}(t) d t\right]^{-1}, \quad i=1,2\end{array}$ \\
\hline (iii) & $\ln \left[\int \alpha_{1}(t) d t\right]-x$ & $\begin{array}{l}{\left[\int \alpha_{1}(t) d t\right]^{-1} F(\xi)} \\
{\left[\int \alpha_{1}(t) d t\right]^{-d} G(\xi)}\end{array}$ & $\begin{array}{l}\beta_{1}(t)=k_{3} \alpha_{1}(t)\left[\int \alpha_{1}(t) d t\right]^{2(d-1)} \\
\alpha_{2}(t)=k_{1} \alpha_{1}(t), \beta_{2}(t)=k_{2} \alpha_{1}(t) \\
\gamma_{i}(t)=\sigma_{i} \alpha_{1}(t)\left[\int \alpha_{1}(t) d t\right]^{-1}, \quad i=1,2\end{array}$ \\
\hline (iv) & $x$ & $\begin{array}{c}F(\xi) \\
\exp \left[-\frac{1}{\mu} \int \alpha_{1}(t) d t\right] G(\xi)\end{array}$ & $\begin{array}{l}\beta_{1}(t)=\frac{k_{3}}{\mu} \alpha_{1}(t) \exp \left[\frac{2}{\mu} \int \alpha_{1}(t) d t\right] \\
\alpha_{2}(t)=k_{1} \alpha_{1}(t), \beta_{2}(t)=k_{2} \alpha_{1}(t) \\
\gamma_{i}(t)=\sigma_{i} \alpha_{1}(t), i=1,2\end{array}$ \\
\hline (v) & $x-\frac{1}{\lambda} \int \alpha_{1}(t) d t$ & $\begin{array}{c}F(\xi) \\
\exp \left[-\frac{1}{\lambda} \int \alpha_{1}(t) d t\right] G(\xi)\end{array}$ & $\begin{array}{l}\beta_{1}(t)=\frac{k_{3}}{\lambda} \alpha_{1}(t) \exp \left[\frac{2}{\lambda} \int \alpha_{1} d t\right] \\
\alpha_{2}(t)=k_{1} \alpha_{1}(t), \beta_{2}(t)=k_{2} \alpha_{1}(t) \\
\gamma_{i}(t)=\sigma_{i} \alpha_{1}(t), i=1,2\end{array}$ \\
\hline (vi) & $x-\int \alpha_{1}(t) d t$ & $F(\xi), G(\xi)$ & $\begin{array}{l}\beta_{1}(t)=k_{3} \alpha_{1}(t), \alpha_{2}(t)=k_{1} \alpha_{1}(t) \\
\beta_{2}(t)=k_{2} \alpha_{1}(t) \\
\gamma_{i}(t)=\sigma_{i} \alpha_{1}(t), i=1,2\end{array}$ \\
\hline (vii) & $x$ & $F(\xi), G(\xi)$ & $\begin{array}{l}\beta_{1}(t)=k_{3}, \alpha_{2}(t)=k_{1} \\
\beta_{2}(t)=k_{2}, \alpha_{1}(t)=k_{o} \\
\gamma_{i}(t)=\sigma_{i}, i=1,2\end{array}$ \\
\hline (viii) & $t$ & $F(\xi), G(\xi)$ & Any arbitrary function of $t$ \\
\hline
\end{tabular}

$k_{o}, k_{1}, k_{2}, k_{3}, \sigma_{1}, \sigma_{2}, a, b, c, d, \mu$, and $\lambda$ are arbitrary constants. In rows (i)-(vi), $\alpha_{1}(t)$ is arbitrary.

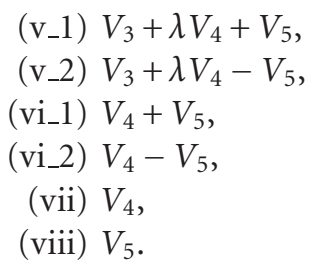

Because the discrete symmetry $(t, x, u, v) \mapsto(t,-x, u, v)$ will map (iii_1), (v_1), and (vi_1) to (iii_2), (v_2), and (vi_2), respectively, in the optimal system we confine ourselves to eight generators only, while neglecting the other three.

In Table 2.1, we now list the similarity variable and the form which we get from (2.11), and also the coefficient functions of the KdV system, for the essential fields in the optimal system. 


\section{The reduced systems and the exact solutions}

In the following, we utilize the similarity variable and the form of the similarity solution, as listed in Table 2.1, to obtain the reduced system of ODEs for (1.1). Some exact solutions for the reduced systems are studied for each type of essential fields mentioned in Table 2.1.

Type (i). The reduced system of ODEs in this case is as follows:

$$
\begin{gathered}
(1+a)\left[\sigma_{1} F^{\prime \prime \prime}+k_{3} G G^{\prime}+F F^{\prime}\right]-\xi F^{\prime}-a F=0 \\
(1+a)\left[\sigma_{2} G^{\prime \prime \prime}+k_{2} F G^{\prime}+k_{1} G F^{\prime}\right]-\xi G^{\prime}-b G=0
\end{gathered}
$$

where prime denotes the differentiation with respect to the variable $\xi$.

To system (3.1) we seek a special solution in the following form:

$$
\begin{aligned}
& F=A_{1} \xi^{-2}+B_{1} \xi \\
& G=A_{2} \xi^{-2}+B_{2} \xi
\end{aligned}
$$

On substituting these expressions for $F$ and $G$ in (3.1), we arrive at the following relations among the various parameters involved:

$$
\begin{aligned}
& B_{1}=\frac{2(1+a)(1+b)-3(2-a)}{(2 b-1)(1+a)+3 k_{2}(2 a-1)(1+a)}, \\
& B_{2}= \pm\left[\frac{B_{1}-B_{1}^{2}}{k_{3}}\right]^{1 / 2}, \quad k_{1}=\frac{(1+b)}{(1+a) B_{1}}-k_{2}, \\
& A_{1}=-\frac{12 \sigma_{2}}{\left(k_{1}+k_{2}\right)}, \quad A_{2}=\frac{\left[(2-a)-(1+a) B_{1}\right]}{k_{3}(1+a) B_{2}} A_{1}, \quad \sigma_{1}=-\frac{\left(A_{1}^{2}+k_{3} A_{2}^{2}\right)}{12 A_{1}},
\end{aligned}
$$

where $a, b, k_{2}, k_{3}$, and $\sigma_{2}$ are arbitrary constants.

Thus, on using the back transformation, we get the solution of the system (1.1) as

$$
\begin{aligned}
& u=A_{1} x^{-2}\left[\int \alpha_{1}(t) d t\right]^{(2-a) /(1+a)}+B_{1} x\left[\int \alpha_{1}(t) d t\right]^{-1} \\
& v=A_{2} x^{-2}\left[\int \alpha_{1}(t) d t\right]^{(2-b) /(1+a)}+B_{2} x\left[\int \alpha_{1}(t) d t\right]^{-(1+b) /(1+a)}
\end{aligned}
$$

Type (ii). The reduced system of ODEs is

$$
\begin{gathered}
\sigma_{1} F^{\prime \prime \prime}+k_{3} G G^{\prime}+F F^{\prime}-F=0, \\
\sigma_{2} G^{\prime \prime \prime}+k_{2} F G^{\prime}+k_{1} G F^{\prime}-c G=0 .
\end{gathered}
$$

Herein, we will look for a special solution in terms of exponential functions, that is, $F=$ $a_{1} \exp (m \xi)$ and $G=a_{2} \exp (m \xi)$, for (3.5). The following exact solution of system (1.1) 
3718 Symmetries and invariant solutions of KdV system

can be readily derived:

$$
\begin{aligned}
& u=a_{1}\left[\int \alpha_{1}(t) d t\right]^{-1} \exp (m x), \\
& v=a_{2}\left[\int \alpha_{1}(t) d t\right]^{-c} \exp (m x),
\end{aligned}
$$

where $a_{1}, a_{2}$, and $m$ are arbitrary constants and

$$
\sigma_{1}=\frac{1}{m^{3}}, \quad k_{3}=-\frac{a_{1}^{2}}{a_{2}^{2}}, \quad k_{2}=-k_{1}, \quad c=\sigma_{2} m^{3} .
$$

Type (iii). In this case, we have

$$
\begin{gathered}
\sigma_{1} F^{\prime \prime \prime}+k_{3} G G^{\prime}+F F^{\prime}-F^{\prime}+F=0, \\
\sigma_{2} G^{\prime \prime \prime}+k_{2} F G^{\prime}+k_{1} G F^{\prime}-G^{\prime}+d G=0 .
\end{gathered}
$$

We employ the hyperbolic tangent method to obtain particular analytic solutions to the system (3.8). On balancing the highest-order derivative terms with the nonlinear terms, one can easily find that the solution will have the form as given by

$$
\begin{aligned}
& F=a_{o}+a_{1} \tanh \xi+a_{2} \tanh ^{2} \xi \\
& G=b_{o}+b_{1} \tanh \xi+b_{2} \tanh ^{2} \xi
\end{aligned}
$$

where $a_{0}, a_{1}, a_{2}$, and $b_{0}, b_{1}, b_{2}$ are constants to be determined. Substituting these forms in (3.8) and performing the algebraic calculations on the relations obtained among various parameters, we get

$$
\begin{gathered}
F=12 \sigma_{1} \operatorname{sech}^{2} \xi, \\
G=-\frac{12 \sigma_{1}}{k_{3} b_{1}}+b_{1} \tanh \xi
\end{gathered}
$$

with

$$
b_{1}^{2}=-\frac{24 \sigma_{1}\left(1-4 \sigma_{1}\right)}{k_{3}}, \quad \sigma_{2}=\frac{1}{4}, \quad k_{2}=\frac{1}{8 \sigma_{1}}, \quad k_{1}=d=0 .
$$

Corresponding solution of system (1.1) can be expressed as follows:

$$
\begin{gathered}
u=12 \sigma_{1} \operatorname{sech}^{2}\left[\ln \left(\int \alpha_{1}(t) d t\right)-x\right]\left[\int \alpha_{1}(t) d t\right]^{-1}, \\
v=-\frac{12 \sigma_{1}}{k_{3} b_{1}}+b_{1} \tanh \left[\ln \left(\int \alpha_{1}(t) d t\right)-x\right] .
\end{gathered}
$$

Type (iv). For the case under consideration the reduced ODEs are

$$
\begin{gathered}
\sigma_{1} F^{\prime \prime \prime}+k_{3} \mu^{-1} G G^{\prime}+F F^{\prime}=0, \\
\sigma_{2} G^{\prime \prime \prime}+k_{2} F G^{\prime}+k_{1} G F^{\prime}-\mu^{-1} G=0 .
\end{gathered}
$$


We are only able to furnish here a linear solution for $F$ and $G$ of the type

$$
\begin{aligned}
& F=\frac{\mu^{-1}}{\left(k_{1}+k_{2}\right)} \xi+a_{2}, \\
& G= \pm\left(\frac{\mu^{-1}}{\left(k_{1}+k_{2}\right) \sqrt{-k_{3} \mu^{-1}}} \xi+\frac{a_{2}}{\sqrt{-k_{3} \mu^{-1}}}\right) .
\end{aligned}
$$

The solution of system (1.1) becomes

$$
\begin{aligned}
& u=\frac{\mu^{-1}}{\left(k_{1}+k_{2}\right)} x+a_{2}, \\
& v= \pm\left(\frac{\mu^{-1}}{\left(k_{1}+k_{2}\right) \sqrt{-k_{3} \mu^{-1}}} x+\frac{a_{2}}{\sqrt{-k_{3} \mu^{-1}}}\right) \exp \left[-\mu^{-1} \int \alpha_{1}(t) d t\right]
\end{aligned}
$$

Type (v). The system of reduced ODEs is given by

$$
\begin{gathered}
\sigma_{1} F^{\prime \prime \prime}+\frac{k_{3}}{\lambda} G G^{\prime}+F F^{\prime}-\frac{1}{\lambda} F^{\prime}=0, \\
\sigma_{2} G^{\prime \prime \prime}+k_{2} F G^{\prime}+k_{1} G F^{\prime}-\frac{1}{\lambda} G^{\prime}-\frac{1}{\lambda} G=0 .
\end{gathered}
$$

Herein, we exploit the idea used in [29] to construct the closed form traveling wave solutions for some nonlinear differential equations. Let us assume that the system (3.16) admits a solution in the form

$$
\begin{aligned}
F & =\sum_{i=0}^{m} a_{i} \phi^{i}+\sum_{i=1}^{m} b_{i} \psi \phi^{i-1}, \\
G & =\sum_{j=0}^{n} c_{j} \phi^{j}+\sum_{j=1}^{n} d_{j} \psi \phi^{j-1},
\end{aligned}
$$

where $\phi$ and $\psi$ are solutions of a coupled Riccati system:

$$
\phi^{\prime}=-k \phi \psi, \quad \psi^{\prime}=k\left(1-\psi^{2}\right),
$$

where $k$ is a constant to be found later. The Riccati system possesses two types of general solutions

$$
\begin{array}{ll}
\phi= \pm \operatorname{sech}(k \xi), & \psi=\tanh (k \xi), \\
\phi= \pm \operatorname{csch}(k \xi), & \psi=\operatorname{coth}(k \xi) .
\end{array}
$$

These two types of solutions satisfy the relations $\psi^{2}=1-\phi^{2}$ and $\psi^{2}=1+\phi^{2}$. We will consider only the first solution for our system. Balancing the highest-order derivative terms with the nonlinear terms in system (3.16) we obtain $m, n=2$. This suggests the following ansatz:

$$
\begin{aligned}
& F=a_{o}+a_{1} \phi+a_{2} \phi^{2}+b_{1} \psi+b_{2} \phi \psi, \\
& G=c_{o}+c_{1} \phi+c_{2} \phi^{2}+d_{1} \psi+d_{2} \phi \psi .
\end{aligned}
$$


Using these in ODEs (3.16), we arrive at a set of algebraic equations which can be solved to get eventually

$$
\begin{aligned}
& F=\frac{1}{\lambda}+\frac{3}{\lambda(1-12 k)} \operatorname{sech}^{2}(k \xi)+\frac{6}{\lambda(1-12 k)} \tanh (k \xi), \\
& G= \pm\left[\frac{45}{\lambda k_{3}(1-12 k)^{2}}\right]^{1 / 2} \operatorname{sech}^{2}(k \xi),
\end{aligned}
$$

subject to the following restrictions on the parameters:

$$
\sigma_{1}=\frac{3}{2 k^{2}(1-12 k) \lambda}, \quad \sigma_{2}=\frac{1}{48 k^{3} \lambda}, \quad k_{2}=\frac{12 k-1}{12 k}, \quad k_{1}=\frac{1-12 k}{6 k} .
$$

Thus, we obtain a very elegant form of an exact analytic periodic solution of the KdV system (1.1)

$$
\begin{aligned}
& u=\frac{1}{\lambda}+\frac{3}{\lambda(1-12 k)} \operatorname{sech}^{2}\left[k\left(x-\frac{1}{\lambda} \int \alpha_{1}(t) d t\right)\right]+\frac{6}{\lambda(1-12 k)} \tanh \left[k\left(x-\frac{1}{\lambda} \int \alpha_{1}(t) d t\right)\right], \\
& v= \pm\left[\frac{45}{\lambda k_{3}(1-12 k)^{2}}\right]^{1 / 2} \operatorname{sech}^{2}\left[k\left(x-\frac{1}{\lambda} \int \alpha_{1}(t) d t\right)\right] \exp \left[-\frac{1}{\lambda} \int \alpha_{1}(t) d t\right] .
\end{aligned}
$$

Type (vi). The Jacobi elliptic function method is used to find an exact solution for the system

$$
\begin{gathered}
\sigma_{1} F^{\prime \prime \prime}+k_{3} G G^{\prime}+F F^{\prime}-F^{\prime}=0, \\
\sigma_{2} G^{\prime \prime \prime}+k_{2} F G^{\prime}+k_{1} G F^{\prime}-G^{\prime}=0 .
\end{gathered}
$$

Following the procedure applied in [15], we take the solution of (3.24) in the form

$$
\begin{aligned}
F & =\sum_{i}^{m} a_{i} s n^{i}(\xi), \\
G & =\sum_{i}^{n} a_{i} s n^{i}(\xi),
\end{aligned}
$$

where $\operatorname{sn}(\xi)$ is the Jacobi elliptic sine function.

We recall first that the Jacobi elliptic cosine function $c n(\xi)$, and the Jacobi elliptic function of third kind $d n(\xi)$, satisfy the following:

$$
\begin{aligned}
& c n^{2}(\xi)=1-s n^{2}(\xi), \quad d n^{2}(\xi)=1-m^{2} s n^{2}(\xi), \\
& \frac{d}{d \xi} s n(\xi)=c n(\xi) d n(\xi), \quad \frac{d}{d \xi} c n(\xi)=-s n(\xi) d n(\xi), \\
& \frac{d}{d \xi} d n(\xi)=-m^{2} c n(\xi) s n(\xi), \quad \text { with modulus } m(0<m<1) .
\end{aligned}
$$


We select the values of $m, n=2$ by balancing the highest-order derivative with the nonlinear term, and this brings in the following ansatz:

$$
\begin{aligned}
& F=a_{o}+a_{1} \operatorname{sn}(\xi)+a_{2} \operatorname{sn}^{2}(\xi), \\
& G=b_{o}+b_{1} \operatorname{sn}(\xi)+b_{2} \operatorname{sn}^{2}(\xi) .
\end{aligned}
$$

Combining (3.24) and (3.27), and performing algebraic computations, we deduce an interesting form of another periodic solution for (1.1) as follows:

$$
\begin{aligned}
& u=F(\xi)=\frac{\left(k_{1}-k_{3} \gamma^{2}\right)+4\left(1+m^{2}\right)\left(k_{1} \sigma_{1}-k_{3} \sigma_{2} \gamma^{2}\right)}{\left(k_{1}-k_{2} k_{3} \gamma^{2}\right)}-\frac{12 \sigma_{2} m^{2}}{\left(k_{1}+k_{2}\right)} s n^{2}(\xi), \\
& v=G(\xi)= \pm\left(\frac{\left(k_{2}-1\right)+4\left(1+m^{2}\right)\left(k_{2} \sigma_{1}-\sigma_{2}\right)}{\left(k_{1} / \gamma-k_{2} k_{3} \gamma\right)}+\frac{12 \sigma_{2} m^{2} \gamma}{\left(k_{1}+k_{2}\right)} s n^{2}(\xi)\right),
\end{aligned}
$$

where $\gamma=\left[\left(\left(k_{1}+k_{2}\right) \sigma_{1}-\sigma_{2}\right) / \sigma_{2} k_{3}\right]^{1 / 2}$ and $\xi=x-\int \alpha_{1}(t) d t$.

It is worth mentioning here that the class of solutions (3.28) gives rise, in particular, to a completely new exact periodic solution for the system analyzed in [32] corresponding to the parameter value $k_{1}=0$.

Type (vii). In this, we follow the procedure in a manner similar to the preceding case to solve the equations

$$
\begin{aligned}
& \sigma_{1} F^{\prime \prime \prime}+k_{3} G G^{\prime}+k_{o} F F^{\prime}=0, \\
& \sigma_{2} G^{\prime \prime \prime}+k_{2} F G^{\prime}+k_{1} G F^{\prime}=0,
\end{aligned}
$$

and the following form of the solution is produced:

$$
\begin{gathered}
F=\frac{4\left(1+m^{2}\right)\left(k_{1} \sigma_{1}-k_{3} \sigma_{2} \gamma^{2}\right)}{\left(k_{o} k_{1}-k_{2} k_{3} \gamma^{2}\right)}-\frac{12 \sigma_{2} m^{2}}{\left(k_{1}+k_{2}\right)} s n^{2}(\xi), \\
G= \pm\left(\frac{4\left(1+m^{2}\right)\left(k_{2} \sigma_{1}-k_{o} \sigma_{2}\right)}{\left(k_{o} k_{1} / \gamma-k_{2} k_{3} \gamma\right)}+\frac{12 \sigma_{2} m^{2} \gamma}{\left(k_{1}+k_{2}\right)} s n^{2}(\xi)\right),
\end{gathered}
$$

where $\gamma=\left[\left(\left(k_{1}+k_{2}\right) \sigma_{1}-k_{o} \sigma_{2}\right) / \sigma_{2} k_{3}\right]^{1 / 2}$. The solution to $(1.1)$ can be expressed as

$$
u=F(\xi), \quad v=G(\xi), \quad \text { with } \xi=x .
$$

Type (viii). The reduced ODEs, in this case are

$$
F^{\prime}=0, \quad G^{\prime}=0 .
$$

The obvious solution of (1.1) is

$$
u=\text { constant }, \quad v=\text { constant } .
$$

\section{Discussion and concluding remarks}

We have investigated the symmetries and invariant solutions of a variable coefficient KdV system. The generalized symmetry method is utilized for the purpose of obtaining the 
group infinitesimals. Using the adjoint action of the symmetry group an optimal system is identified. The basic fields of the optimal system lead to reductions that are inequivalent with respect to the symmetry transformations. For each element in the optimal system, some exact solutions are attempted for the KdV system. It is important to note that, besides deriving the exact solutions for the system under investigation, new exact solutions have also been deduced in particular, for a system examined in [32]. Moreover, in one instance (refer to (3.28)), the solution furnished brings forth a new class of exact periodic solutions of the same system [32]. In almost all the cases, one can choose the arbitrary function $\alpha_{1}(t)$, along with various other arbitrary parameters, in a suitable manner, and this provides enough freedom to build solutions that may correspond to a particular physical situation. It is to be mentioned that, with the aid of Maple, the exact solutions reported here in this paper are found to indeed satisfy the KdV system. Finally, it is being pointed out that the KdV system examined in the present study will also admit some additional symmetries for the special case $\alpha_{1}(t)=\beta_{2}(t)$. The work is in progress and will be communicated later upon completion.

The main results of Sections 2 and 3 are summarized as follows.

THEOREM 4.1. For a given smooth function $\alpha_{1}(t)$, the KdV system (1.1) remains invariant under a 5-parameter Lie algebra spanned by the following basis elements:

$$
\begin{aligned}
& V_{1}=\left[\frac{1}{\alpha_{1}(t)} \int \alpha_{1}(t) d t\right] \frac{\partial}{\partial t}+x \frac{\partial}{\partial x}, \\
& V_{2}=\left[\frac{1}{\alpha_{1}(t)} \int \alpha_{1}(t) d t\right] \frac{\partial}{\partial t}-u \frac{\partial}{\partial u}, \\
& V_{3}=-v \frac{\partial}{\partial v}, \quad V_{4}=\frac{1}{\alpha_{1}(t)} \frac{\partial}{\partial t}, \quad V_{5}=\frac{\partial}{\partial x} .
\end{aligned}
$$

The admissible forms of the coefficient functions satisfy the following equations:

$$
\begin{gathered}
\frac{d}{d t}\left[\frac{\beta_{1}(t)}{\alpha_{1}(t)}\left\{\left(a_{1}+a_{2}\right) \int \alpha_{1}(t) d t+a_{4}\right\}\right]+\left(a_{2}-2 a_{3}-a_{1}\right) \beta_{1}(t)=0, \\
\frac{d}{d t}\left[\frac{\gamma_{i}(t)}{\alpha_{1}(t)}\left\{\left(a_{1}+a_{2}\right) \int \alpha_{1}(t) d t+a_{4}\right\}\right]-3 a_{1} \gamma_{i}(t)=0, \quad i=1,2, \\
\alpha_{2}(t)=k_{1} \alpha_{1}(t), \quad \beta_{2}(t)=k_{2} \alpha_{1}(t),
\end{gathered}
$$

where $a_{1}, a_{2}, a_{3}, a_{4}, k_{1}$, and $k_{2}$ are arbitrary constants.

THEOREM 4.2 (periodic solution). Suppose that the coefficient functions are as given in row (v) of Table 2.1. Then

$$
\begin{aligned}
& u=\frac{1}{\lambda}+\frac{3}{\lambda(1-12 k)} \operatorname{sech}^{2}\left[k\left(x-\frac{1}{\lambda} \int \alpha_{1}(t) d t\right)\right]+\frac{6}{\lambda(1-12 k)} \tanh \left[k\left(x-\frac{1}{\lambda} \int \alpha_{1}(t) d t\right)\right], \\
& v= \pm\left[\frac{45}{\lambda k_{3}(1-12 k)^{2}}\right]^{1 / 2} \operatorname{sech}^{2}\left[k\left(x-\frac{1}{\lambda} \int \alpha_{1}(t) d t\right)\right] \exp \left[-\frac{1}{\lambda} \int \alpha_{1}(t) d t\right]
\end{aligned}
$$


form a solution to the KdV equations (1.1), when

$$
\sigma_{1}=\frac{3}{2 k^{2}(1-12 k) \lambda}, \quad \sigma_{2}=\frac{1}{48 k^{3} \lambda}, \quad k_{2}=\frac{12 k-1}{12 k}, \quad k_{1}=\frac{1-12 k}{6 k} ;
$$

$\lambda \neq 0, k_{3} \neq 0, k \neq 1 / 12$ are arbitrary constants; and $\alpha_{1}(t)$ is an arbitrary smooth function.

THEOREM 4.3 (periodic solution). Suppose that the coefficient functions are as given in row (vi) of Table 2.1. Then

$$
\begin{aligned}
& u=F(\xi)=\frac{\left(k_{1}-k_{3} \gamma^{2}\right)+4\left(1+m^{2}\right)\left(k_{1} \sigma_{1}-k_{3} \sigma_{2} \gamma^{2}\right)}{\left(k_{1}-k_{2} k_{3} \gamma^{2}\right)}-\frac{12 \sigma_{2} m^{2}}{\left(k_{1}+k_{2}\right)} s n^{2}(\xi), \\
& v=G(\xi)= \pm\left(\frac{\left(k_{2}-1\right)+4\left(1+m^{2}\right)\left(k_{2} \sigma_{1}-\sigma_{2}\right)}{\left(k_{1} / \gamma-k_{2} k_{3} \gamma\right)}+\frac{12 \sigma_{2} m^{2} \gamma}{\left(k_{1}+k_{2}\right)} s n^{2}(\xi)\right),
\end{aligned}
$$

where $\gamma=\left[\left(\left(k_{1}+k_{2}\right) \sigma_{1}-\sigma_{2}\right) / \sigma_{2} k_{3}\right]^{1 / 2} \neq 0, k_{1} \neq k_{2} k_{3} \gamma^{2}, 0<m<1, \sigma_{2} \neq 0, k_{3} \neq 0$, and $k_{1} \neq-k_{2}$ are all arbitrary constants and $\xi=x-\int \alpha_{1}(t) d t$, with $\alpha_{1}(t)$ as a smooth arbitrary function, is a solution of the $\mathrm{KdV}$ system (1.1).

Theorem 4.4. Suppose that the coefficient functions are as given in row (i) of Table 2.1. Then, for an arbitrary smooth function $\alpha_{1}(t)$, the KdV system (1.1) has a solution of the form

$$
\begin{aligned}
& u=A_{1} x^{-2}\left[\int \alpha_{1}(t) d t\right]^{(2-a) /(1+a)}+B_{1} x\left[\int \alpha_{1}(t) d t\right]^{-1}, \\
& v=A_{2} x^{-2}\left[\int \alpha_{1}(t) d t\right]^{(2-b) /(1+a)}+B_{2} x\left[\int \alpha_{1}(t) d t\right]^{-(1+b) /(1+a)},
\end{aligned}
$$

where

$$
\begin{aligned}
& B_{1}=\frac{2(1+a)(1+b)-3(2-a)}{(2 b-1)(1+a)+3 k_{2}(2 a-1)(1+a)}, \\
& B_{2}= \pm\left[\frac{B_{1}-B_{1}^{2}}{k_{3}}\right]^{1 / 2}, \quad A_{1}=-\frac{12 \sigma_{2}}{\left(k_{1}+k_{2}\right)}, \quad A_{2}=\frac{\left[(2-a)-(1+a) B_{1}\right]}{k_{3}(1+a) B_{2}} A_{1}, \\
& k_{1}=\frac{(1+b)}{(1+a) B_{1}}-k_{2}, \quad \sigma_{1}=-\frac{\left(A_{1}^{2}+k_{3} A_{2}^{2}\right)}{12 A_{1}}
\end{aligned}
$$

with $a, b, k_{2}, k_{3}$, and $\sigma_{2}$ as arbitrary constants.

THEOREM 4.5. Suppose that the coefficient functions are as given in row (iii) of Table 2.1. Then, for an arbitrary smooth function $\alpha_{1}(t)$, the KdV system (1.1) has a solution of the form

$$
\begin{aligned}
& u=12 \sigma_{1} \operatorname{sech}^{2}\left[\ln \left(\int \alpha_{1}(t) d t\right)-x\right]\left[\int \alpha_{1}(t) d t\right]^{-1}, \\
& v=-\frac{12 \sigma_{1}}{k_{3} b_{1}}+b_{1} \tanh \left[\ln \left(\int \alpha_{1}(t) d t\right)-x\right]
\end{aligned}
$$


where

$$
b_{1}^{2}=-\frac{24 \sigma_{1}\left(1-4 \sigma_{1}\right)}{k_{3}}, \quad \sigma_{2}=\frac{1}{4}, \quad k_{2}=\frac{1}{8 \sigma_{1}}, \quad k_{1}=d=0
$$

with $\sigma_{1}, k_{3}$ as arbitrary constants.

\section{Acknowledgments}

The authors would like to express their sincere thanks to the referee for his useful and constructive suggestions. R. K. Gupta gratefully acknowledges the financial support provided by JUIT, Waknaghat, for this work.

\section{References}

[1] I. Sh. Akhatov, R. K. Gazizov, and N. H. Ibragimov, Group classification of equations of nonlinear filtration, Soviet Math. Dokl. 35 (1987), 384-386.

[2] D. J. Arrigo, J. M. Hill, and P. Broadbridge, Nonclassical symmetry reductions of the linear diffusion equation with a nonlinear source, IMA J. Appl. Math. 52 (1994), no. 1, 1-24.

[3] O. P. Bhutani and P. Mital, Similarity solutions of the equations of dynamical meteorology via symmetry method, J. Meteorol. Soc. Jpn 64 (1986), 593-598.

[4] O. P. Bhutani and K. Singh, Generalized similarity solutions for the type D fluid in fivedimensional flat space, J. Math. Phys. 39 (1998), no. 6, 3203-3212.

[5] O. P. Bhutani, K. Singh, and D. K. Kalra, On certain classes of exact solutions of Einstein equations for rotating fields in conventional and nonconventional form, Internat. J. Engrg. Sci. 41 (2003), no. 7, 769-786.

[6] G. W. Bluman and J. D. Cole, Similarity Methods for Differential Equations, Applied Mathematical Sciences, vol. 13, Springer, New York, 1974.

[7] G. W. Bluman and S. Kumei, Symmetries and Differential Equations, Applied Mathematical Sciences, vol. 81, Springer, New York, 1989.

[8] G. W. Bluman, G. J. Reid, and S. Kumei, New classes of symmetries for partial differential equations, J. Math. Phys. 29 (1988), no. 4, 806-811.

[9] F. Cariello and M. Tabor, Painlevé expansions for nonintegrable evolution equations, Phys. D 39 (1989), no. 1, 77-94.

[10] K.-S. Chou and C. Z. Qu, Symmetry groups and separation of variables of a class of nonlinear diffusion-convection equations, J. Phys. A 32 (1999), no. 35, 6271-6286.

[11] P. A. Clarkson, New similarity solutions for the modified Boussinesq equation, J. Phys. A 22 (1989), no. 13, 2355-2367.

[12] P. A. Clarkson and M. D. Kruskal, New similarity reductions of the Boussinesq equation, J. Math. Phys. 30 (1989), no. 10, 2201-2213.

[13] P. A. Clarkson and E. L. Mansfield, Symmetry reductions and exact solutions of a class of nonlinear heat equations, Phys. D 70 (1994), no. 3, 250-288.

[14] A. S. Fokas and Q. M. Liu, Nonlinear interaction of traveling waves of nonintegrable equations, Phys. Rev. Lett. 72 (1994), no. 21, 3293-3296.

[15] Z. Fu, S. K. Liu, S. D. Liu, and Q. Zhao, New Jacobi elliptic function expansion and new periodic solutions of nonlinear wave equations, Phys. Lett. A 290 (2001), no. 1-2, 72-76.

[16] J. M. Hill, A. J. Avagliano, and M. P. Edwards, Some exact results for nonlinear diffusion with absorption, IMA J. Appl. Math. 48 (1992), no. 3, 283-304.

[17] S. Hood, On direct implicit reductions of a nonlinear diffusion equation with an arbitrary function-generalizations of Clarkson's and Kruskal's method, IMA J. Appl. Math. 64 (2000), no. 3, 223-244. 
[18] J. R. King, Exact similarity solutions to some nonlinear diffusion equations, J. Phys. A 23 (1990), no. $16,3681-3697$.

[19] Some non-self-similar solutions to a nonlinear diffusion equation, J. Phys. A 25 (1992), no. $18,4861-4868$.

[20] - Exact polynomial solutions to some nonlinear diffusion equations, Phys. D 64 (1993), no. $1-3,35-65$.

[21] S.-Y. Lou, Symmetry analysis and exact solutions of the $2+1$ dimensional sine-Gordon system, J. Math. Phys. 41 (2000), no. 9, 6509-6524.

[22] S.-Y. Lou, X.-Y. Tang, and J. Lin, Similarity and conditional similarity reductions of a $(2+1)$ dimensional KdV equation via a direct method, J. Math. Phys. 41 (2000), no. 12, 8286-8303.

[23] M. H. M. Moussa, Similarity solutions to non-linear partial differential equation of physical phenomena represented by the Zakharov-Kuznetsov equation, Internat. J. Engrg. Sci. 39 (2001), no. $14,1565-1575$.

[24] A. C. Newell, M. Tabor, and Y. B. Zeng, A unified approach to Painlevé expansions, Phys. D 29 (1987), no. 1-2, 1-68.

[25] M. C. Nucci, Iterating the nonclassical symmetries method, Phys. D 78 (1994), no. 1-2, 124-134.

[26] P. J. Olver, Applications of Lie Groups to Differential Equations, 2nd ed., Graduate Texts in Mathematics, vol. 107, Springer, New York, 1993.

[27] C. Z. Qu, S. L. Zhang, and R. Liu, Separation of variables and exact solutions to quasilinear diffusion equations with nonlinear source, Phys. D 144 (2000), no. 1-2, 97-123.

[28] L. Yang, K. Yang, and H. Luo, Complex version KdV equation and the periods solution, Phys. Lett. A 267 (2000), no. 5-6, 331-334.

[29] R.-X. Yao and Z.-B. Li, New exact solutions for three nonlinear evolution equations, Phys. Lett. A 297 (2002), no. 3-4, 196-204.

[30] S. L. Zhang, S.-Y. Lou, and C. Z. Qu, Variable separation and exact solutions to generalized nonlinear diffusion equations, Chinese Phys. Lett. 19 (2002), no. 12, 1741-1744.

[31] R. Z. Zhdanov, Conditional Lie-Bäcklund symmetry and reduction of evolution equations, J. Phys. A 28 (1995), no. 13, 3841-3850.

[32] Y. Zhou, M. Wang, and Y. Wang, Periodic wave solutions to a coupled KdV equations with variable coefficients, Phys. Lett. A 308 (2003), no. 1, 31-36.

K. Singh: Department of Mathematics, Jaypee University of Information Technology, Waknaghat, P.O. Dumehar Bani, Kandaghat, Distt. Solan, Pin-173215 (H.P.), India

E-mail addresses: karan_jeet@yahoo.com; ksingh_juit@hotmail.com

R. K. Gupta: Department of Mathematics, Jaypee University of Information Technology, Waknaghat, P.O. Dumehar Bani, Kandaghat, Distt. Solan, Pin-173215 (H.P.), India

E-mail address: rajeshateli@rediffmail.com 


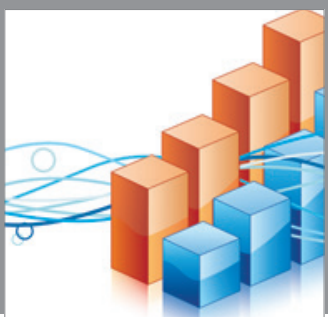

Advances in

Operations Research

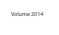

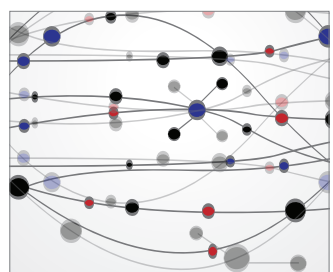

\section{The Scientific} World Journal
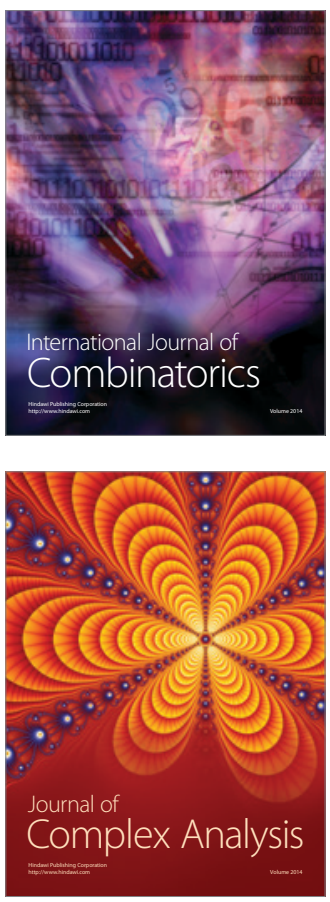

International Journal of

Mathematics and

Mathematical

Sciences
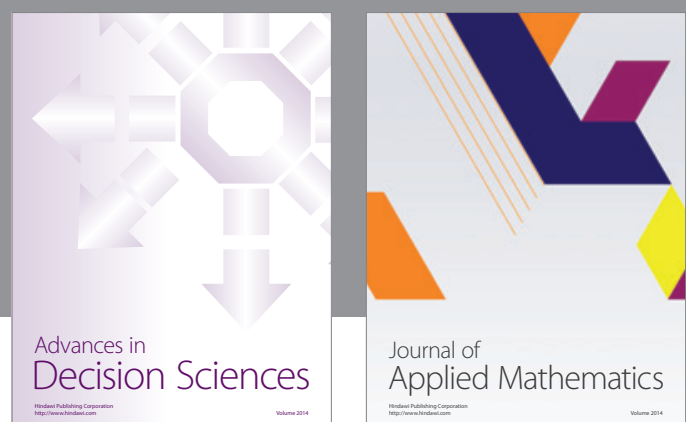

Journal of

Applied Mathematics
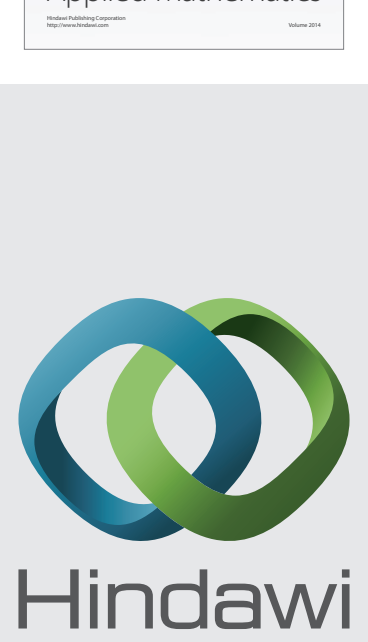

Submit your manuscripts at http://www.hindawi.com
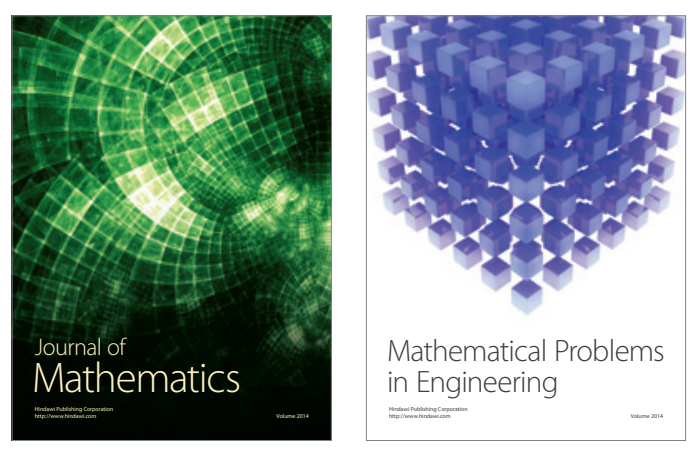

Mathematical Problems in Engineering
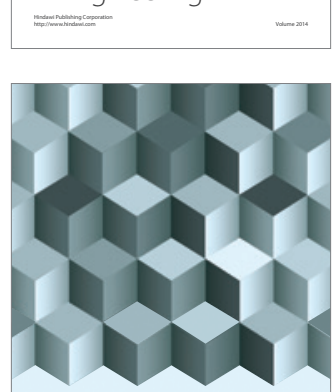

Journal of

Function Spaces
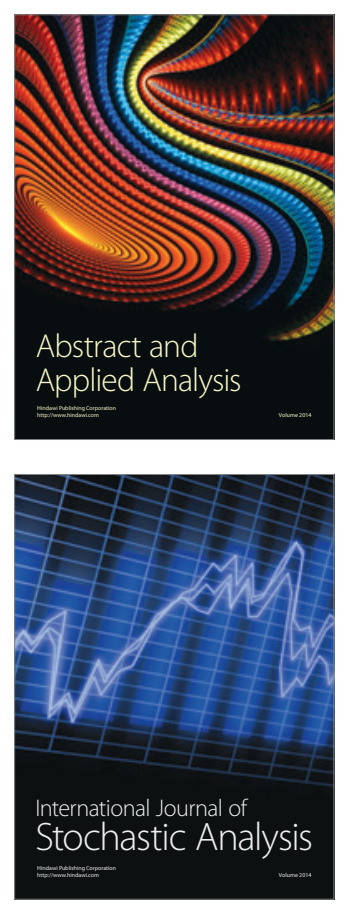

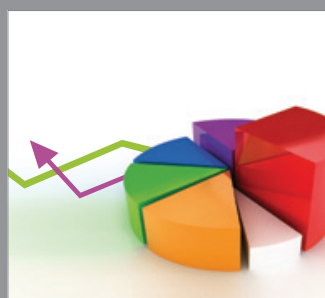

ournal of

Probability and Statistics

Promensencen
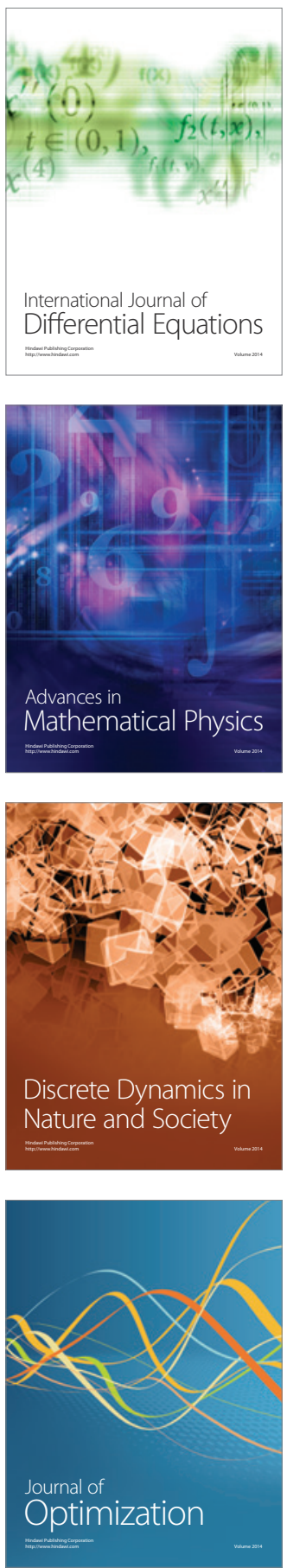